\title{
Morfoanatomía e histoquímica de Hypericum juniperinum (Hypericaceae)
}

\author{
Laura Alejandra Mejía-Agudelo ${ }^{1}$, Juan Camilo Marín-Loaiza ${ }^{1}$, \& Xavier Marquínez-Casas ${ }^{2 *}$ \\ 1. Universidad Nacional de Colombia-Sede Bogotá, Facultad de Ciencias, Carrera 30 \# 45-03, Bogotá D.C., 111321, \\ Colombia; ${ }^{1}$ Departamento de Farmacia, GIFFUN, Ed 450, Laboratorio 303; laamejiaag@unal.edu.co, \\ jcmarinlo@unal.edu.co \\ 2. Departamento de Biología, SISBIO (COL0064669), Ed 421, Laboratorio 112; xmarquinezc@unal.edu.co \\ $*$ Correspondence
}

Recibido 02-I-2019. Corregido 10-VI-2019. Aceptado 06-IX-2019.

\begin{abstract}
Morphoanatomy and histochemical characterization of Hypericum juniperinum (Hypericaceae). Hypericum is a genus that belongs to the Hypericaceae family, with has 469 species of herbs, trees and shrubs. Two sections in particular, Brathys and Trygynobrathys are an integral part of the Paramo flora. Hypericum genus has a wide variety of metabolites that are associated with multiple pharmacological activities, and specifically in $H$. juniperinum antidepressant properties related to the presence of flavonoids and phenolic compounds have been reported. The objective of this research is to relate the anatomical structures with the metabolites detected by histochemical methods present in the vegetative and reproductive organs of Hypericum juniperinum collected in the Colombian Paramo. The aerial organs (stems, leaves and flowers) of Hypericum juniperinum were collected in the village Arbolocos of the municipality of Cuitiva, Boyacá (voucher code No. 589611, COL). For the anatomical analysis, the organs of $H$. juniperinum were processed with fixation techniques, incorporation in paraffin, cutting in microtome and staining with Astra-blue and basic fuchsin; for the histochemical analysis freehand cuts were made with specific stains for lipids, phenol compounds, alkaloids and pectins. The anatomy of the organs of H. juniperinum should be understood in the context of the living conditions of the Paramo. Leaves are highly xeromorphic as they are chartaceous, rosette-shaped, convolute and upright, with parenchyma in bistratified palisade, thickened walls, thick cuticle and epicuticular wax in both foliar epidermis, stomata on the abaxial side and with epidermal chamber. However, it has some mesomorphic characteristics such as parenchymatic bundle sheath and loose spongy parenchyma. Hypericum juniperinum has translucent glands in leaves, calyx and gynoecium, and lacks black glands typical of the Hypericum section belonging to the medicinal plant Hypericum perforatum. Histochemical analysis showed lipids in leaf canals, radial parenchyma of the stem and in some floral structures. Phenolic compounds were detected along the leaf, in the phloem of the stem and in the anthers. Hypericum juniperinum lacks alkaloids and presents pectins in the parenchyma of the sheath surrounding the vascular bundle of the leaf. The present work contributes by relating the metabolites with their distribution in the tissues of this native species. In addition, the anatomy of H. juniperinum has characteristics of the Paramo plants. Description given here could be used as a preliminary monograph of plants from the Hypericum genus with promising therapeutic properties.
\end{abstract}

Key words: anatomical characterization; phenolic compounds; histochemistry; translucent glands; canals.

Mejía-Agudelo, L. A., Marín-Loaiza, J. C., \& Marquínez-Casas, X. (2019). Morfoanatomía e histoquímica de Hypericum juniperinum (Hypericaceae). Revista de Biología Tropical, 67(6), 1160-1169.

Hypericum pertenece a la familia Hypericaceae y comprende aproximadamente 469 especies de hierbas, árboles y arbustos distribuidos en todos los climas templados del mundo; sin embargo, en los trópicos sólo están presentes en el rango altitudinal comprendido entre 1900 y 3600 msnm (Ernst, 2003). Actualmente las especies de Hypericum se 
agrupan en 36 secciones de acuerdo con su morfología, distribución y citología (Nürk, 2011). La mayoría de las especies nativas de regiones montañosas de Centroamérica y Sudamérica forman parte integral del tipo de vegetación de páramo y subpáramo, y pertenecen a la sección Brathys (Crockett, Eberhardt, Kunert, \& Schübly, 2010), destacándose en Colombia las especies $H$. goyanesii, H. juniperinum, H. mexicanum y H. strictum (Marín \& Parra, 2015). Hypericum posee una amplia diversidad de metabolitos que se asocian con múltiples efectos farmacológicos. Propiedades de tipo antidepresivo (hiperforina, adhiperforina y flavonoides), antiviral (hipericina y pseudohipericina), antiinflamatorio, espasmolítica (flavonoides) y antimicrobiano han sido descritas para especies de este género (Greeson, Sanford, \& Monti, 2001; Ernst, 2003; Saddiqe, Naeem, \& Maimoona, 2010; Stojanovic, Dordevic, \& Smelcerovic, 2013). Los metabolitos a los que se les atribuye dichas actividades están almacenados en estructuras de secreción presentes, tanto en los órganos vegetativos como en los reproductivos (Perrone, De Rosa, De Castro, \& Colombo, 2013), y dentro de tales metabolitos se encuentran hipericina e hiperforina, que presentan un rol defensivo, aumentando sus niveles después del ataque de herbívoros generalistas (Sirvent, Krasnoff, \& Gibson, 2003).

Debido a la importancia taxonómica de las estructuras de secreción presentes en el género, Green (1884) realizó una revisión de las descripciones anatómicas de Hypericum y la complementó con observaciones de Hypericum calycinum, $H$. tetrapterum, $H$. hyrsutum, у $H$. perforatum, encontrando que hay cuatro tipos de estructuras secretoras: (1) cavidades lisígenas, translúcidas con aceites, (2) canales de resina densos, en corteza y médula, (3) canales esquizógenos delgados en el floema y (4) nódulos o glándulas negras en hojas y flores.

Posteriormente se ha establecido que el origen de todas las estructuras secretoras de este género es esquizógeno; por lo tanto, estas categorías han sido revaluadas. En este contexto, Curtis y Lersten (1990) describen que en las hojas y flores de $H$. perforatum y $H$. balearicum existen cavidades esquizógenas con aceite y glándulas negras, definidas como nódulos carentes de espacios intercelulares, rodeados por una vaina de células aplanadas, que no presentan contenido resinoso. Ciccarelli, Andreucci y Pagni (2001a y 2001b) y Lotocka y Osinska (2010) definen finalmente, tres estructuras de secreción: las glándulas translúcidas, las glándulas negras y los canales secretores, que pueden ser de tres tipos A, B y C.

$H$. juniperinum, es conocido comúnmente como chite o guardarocio y tradicionalmente se emplean las flores para tratar la tos y las ramas para hacer escobas o fuego (Duarte \& Parra, 2015). Recientemente se han detectado en esta especie flavonoides y compuestos fenólicos y se ha reportado actividad de tipo antidepresivo del extracto metanólico y las fracciones de acetato de etilo y butanol en ratones (MejíaAgudelo et al., 2019). En esta investigación se realizó el estudio morfoanatómico e histoquímico de H. Juniperum con el fin de relacionar los metabolitos producidos con las estructuras anatómicas presentes en los órganos vegetativos y reproductivos La descripción realizada aquí puede ser empleada para elaborar una monografía de esta especie andina.

\section{MATERIALES Y MÉTODOS}

Material vegetal: Los órganos aéreos (tallos, hojas y flores) de Hypericum juniperinum (Kunth) fueron recolectados en la vereda Arbolocos del municipio de Cuítiva, Boyacá, Colombia. Un ejemplar fue depositado en el Herbario Nacional Colombiano (COL) con código 589611.

Histología de los órganos aéreos: Los órganos aéreos (tallos, hojas y flores) de Hypericum juniperinum fueron fijados en una solución de FAA (10:5:85, formol: ácido acético: etanol $70 \%$ ) por $48 \mathrm{~h}$ y posteriormente fueron tratados con los protocolos convencionales de Johansen (1940) modificados, siguiendo la metodología descrita por Robles, Raz y Marquínez (2016). Al final se adicionó citoresina 
sobre los portaobjetos, se montó el cubreobjetos y se dejó secar al aire durante 2 semanas. Adicionalmente, la descripción de las estructuras secretoras de esta especie se realizó de acuerdo a Ciccarelli et al., (2001a).

Histoquímica de los órganos aéreos: $\mathrm{Se}$ seccionaron a mano alzada tallos, hojas y flores recién recolectados de H. Juniperinum y se realizaron tinciones histoquímicas diferenciales: Fluoroglucinol-HCl para lignina, rojo de rutenio para pectinas, Sudán III, IV y Sudán negro para grasas, aceites, suberina y cutina (Ruzin, 1999), reactivo de Dragendorff para alcaloides (Brechu-Franco, Laguna-Hernández, Dela-Cruz-Chacón, \& González-Esquinca, 2016) y cloruro férrico para compuestos fenólicos (Texeira \& Monteiro, 2017). En todas las pruebas se compararon las tinciones con una sección no teñida (blanco), con el fin de descartar falsos positivos.

Fotografía y procesamiento de imágenes: El material fresco, las tinciones histoquímicas y los micropreparados fueron observados y fotografiados utilizando un estereoscopio Leica M-205 en modo multifocus con cámara MC170HD, y un microscopio Olympus BX50 con cámara Moticam-pro 28. Las imágenes digitales fueron procesadas con los programas Adobe Photoshop CC y Adobe Indesign.

\section{RESULTADOS}

Descripción general: Hypericum juniperinum es un arbusto con hojas sésiles, erectas e imbricadas, la lámina foliar es acicular y con ápice agudo, con glándulas redondeadas visibles a simple vista (Fig. 1A). Las flores son terminales, agrupadas en pares y conformadas por cuatro verticilos: cáliz conformado por cinco sépalos verdes lanceolados y libres, corola con cinco pétalos libres, oblongos y de color amarillo intenso, androceo con aproximadamente 21 estambres fasciculados, agrupados en grupos de 4-5 estambres de color amarillo y filamentos alargados, y gineceo ovoide, sincárpico, tricarpelar, trilocular en la base y unilocular en el ápice, donde se localizan 3 a 5 estilos que rematan en estigmas glandulares y con forma de cabezuela (Fig. 1B).

Anatomía e histoquímica del tallo: Se estudiaron tallos jóvenes (Fig. 1C) y maduros (Fig. 1D). En tallos maduros se presenta un súber prominente, compuesto por varias capas de células rectangulares, con paredes gruesas suberificadas y contenido de color café, felógeno uniestratificado. En todos los tallos se observa una delgada corteza parenquimática conformada con células de contenido variable. En la corteza y el floema se presentan ductos rodeados por cuatro a cinco células secretoras (cnA: canales tipo A) (Fig. 1C). El floema presenta radios unicelulares dilatados, alternados con agrupaciones de elementos de tubo criboso y células acompañantes de tamaño pequeño, la zona cambial se reduce a dos o tres capas de células. El xilema presenta radios unicelulares o rara vez bicelulares, conformados por parénquima lignificado, entre los radios se presentan grandes vasos, sin contenido y de paredes más delgadas, uniperforados y con punteaduras reticuladas en sección longitudinal. El parénquima paratraqueal presenta contenido denso y en algunos casos oleoso, las fibras son escasas. No se observaron anillos de crecimiento (Fig. 1E).

En el análisis histoquímico, Sudán III, IV y negro reaccionan con la suberina del tallo y con aceites presentes en los canales de corteza y floema, dando una coloración roja para las dos primeras tinciones (Fig. 1F) y negra en la última (Fig. 1G). El cloruro férrico genera una coloración negra continua en el floema y en la médula del tallo asociada a la presencia de sustancias de tipo fenólico (Fig. 1H). La tinción con fluoroglucinol ácido genera una coloración rosada-roja intensa en el xilema del tallo, siendo positiva para paredes lignificadas (Fig. 1I). La prueba de Dragendorff fue negativa, lo que indica ausencia de alcaloides. La prueba con rojo de rutenio indica la presencia ocasional de pectinas en células del xilema.

Hoja: Las hojas de $H$. juniperinum se encuentran dispuestas alternamente en forma 

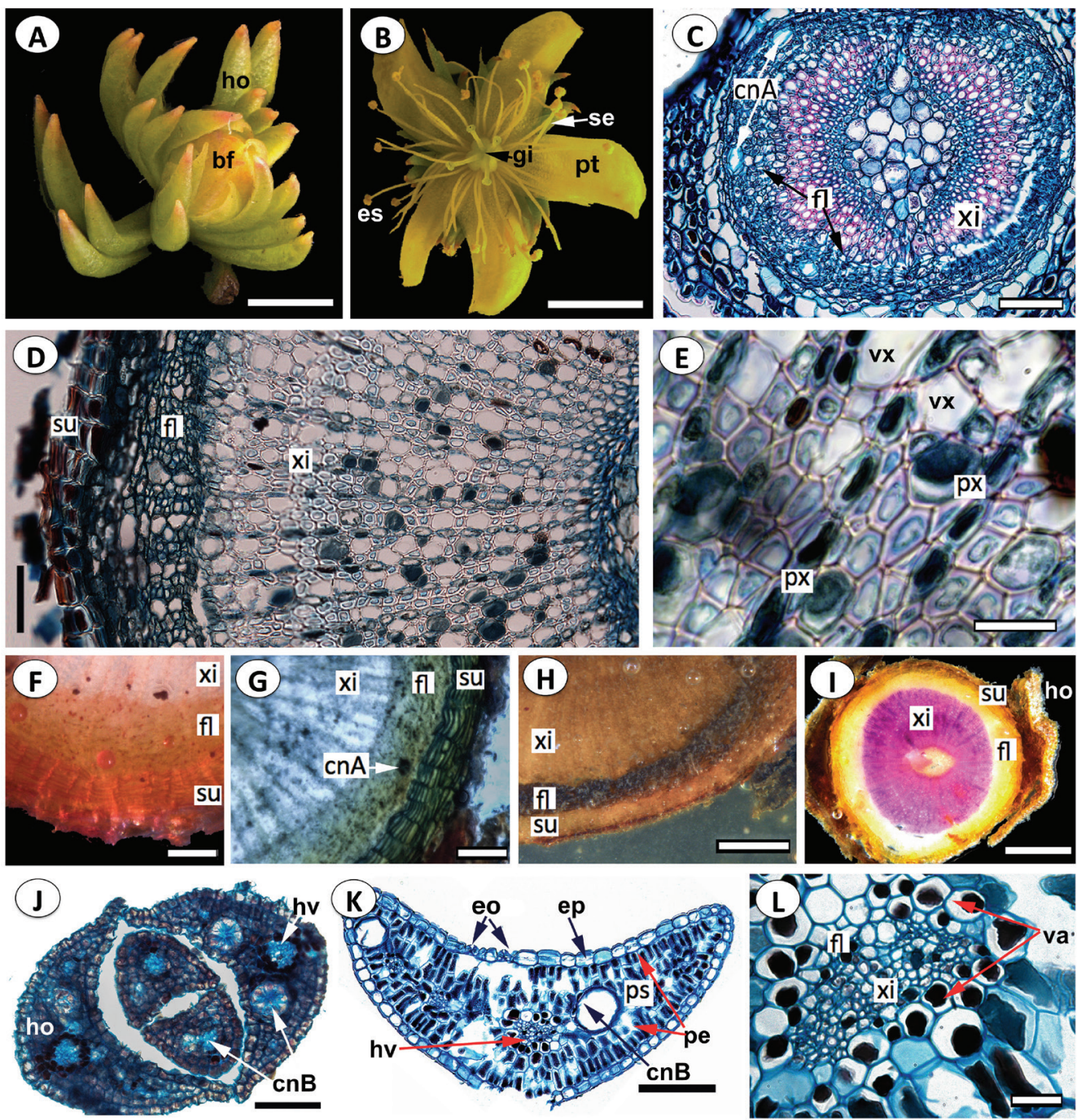

Fig. 1. Morfología floral y anatomía e histoquímica de tallos y hojas de Hypericum juniperinum. A. Hábito general del ápice del tallo; B. flor; C. Sección transversal (ST) de tallo joven; D. ST de tallo maduro. E. Detalle del xilema; F-I. Pruebas histoquímicas en tallo maduro; F. Sudán IV; G. Sudán Black; H. Cloruro férrico, positiva en floema; I. Fluoroglucinol; J. Disposición de las hojas jóvenes; K. ST de hoja madura; L. Detalle del haz vascular de la hoja. Bf = botón floral, cnA $=$ canales tipo $\mathrm{A}, \mathrm{cnB}=$ canales tipo $\mathrm{B}$, ep = epidermis, es = estambre, eo = estomas, $\mathrm{fl}=$ floema, $\mathrm{gi}=$ gineceo, $\mathrm{hv}=\mathrm{haz}$ vascular, ho $=$ hoja, $\mathrm{pe}=$ parénquima empalizada, $\mathrm{ps}=$ parénquima esponjoso, $\mathrm{pt}=$ pétalo, $\mathrm{px}=$ parénquima xilemático, $\mathrm{se}$ = sépalo, $\mathrm{su}=$ súber, va = vaina haz vascular, vx = vasos xilemáticos, xi = xilema. A-B, F-I. Estereomicroscopio; C-E, J-L. Microscopio óptico, tinción con Azul de Astra-Fucsina básica. Escalas: $2 \mathrm{~mm}$ en A., $1 \mathrm{~mm}$ en B., $500 \mathrm{~mm}$ en H-I., $100 \mathrm{~mm}$ en F-G., J-K., $50 \mathrm{~mm}$ en C-D., $20 \mathrm{~mm}$ en E,L. Figuras a color en la versión digital.

Fig. 1. Floral morphology, anatomy and histochemistry of stems and leaves of Hypericum juniperinum. A. General habit of the apex of the stem; B. Flower; C. Cross section (ST) of young stem; D. ST of mature stem. E. Detail of the xylem; F-I. Histochemical tests on mature stem; F. Sudan IV; G. Sudan Black; H. Ferric chloride, positive in phloem; I. Fluoroglucinol; J. Arrangement of young leaves; K. ST of mature leaf; L. Detail of the vascular bundle of the leaf. Bf $=$ floral button, $\mathrm{cnA}$ $=$ type $\mathrm{A}$ channels, $\mathrm{cnB}=$ type $\mathrm{B}$ channels, ep = epidermis, es = stamen, eo = stomata, $\mathrm{fl}=$ phloem, $\mathrm{gi}=$ gynoecium, $\mathrm{hv}=$ vascular bundle, ho $=$ leaf, $\mathrm{pe}=$ palisade parenchyma, $\mathrm{ps}=$ spongy parenchyma, $\mathrm{pt}=$ petal, $\mathrm{px}=\mathrm{xylematic}$ parenchyma, $\mathrm{se}$ = sepal, su $=$ suber, va $=$ vascular bundle sheath, vx $=$ xylematic vessels, $x i=$ xylem. A-B, F-I. Stereomicroscope; C-E, J-L. Optical microscope, staining with astra blue- basic fuchsine. Scales: $2 \mathrm{~mm}$ in A., $1 \mathrm{~mm}$ in B., $500 \mu \mathrm{m}$ in H-I., $100 \mu \mathrm{m}$ in F-G., J-K., $50 \mu \mathrm{m}$ in C-D., $20 \mu \mathrm{m}$ in E., L. Colored figures in the digital version. 
de roseta. Presentan una epidermis conformada por células de recubrimiento redondeadas con cutícula externa gruesa y estomas en la cara adaxial (hoja epistomática) y de tipo anomocítico (Fig. 1J, Fig. 1K), no se observaron tricomas. El mesófilo está conformado en su mayor parte por dos capas de parénquima en empalizada, tanto en la superficie adaxial como abaxial, en el centro se presenta un parénquima más laxo (esponjoso) entre el haz vascular central mayor y los dos haces menores. Los haces están rodeados de una vaina con contenido tanífero (Fig. 1L), adicionalmente la lámina foliar presenta canales esquizógenos de tipo $\mathrm{B}$ rodeados por $8-12$ células de parénquima secretor (Fig. 1J).

En la evaluación histoquímica se observan sustancias de carácter lipídico en la cutícula de la epidermis, al interior de los canales esquizógenos y en algunas células parenquimáticas; reveladas por las pruebas con el reactivo de Sudán (Fig. 2A). La prueba de cloruro férrico generó una coloración negra distribuida en todos los tejidos de la hoja, exceptuando los canales, evidenciando sustancias de tipo fenólico. El fluoroglucinol revela únicamente la lignina del xilema en los haces vasculares (Fig. 2B), no se presenta esclerénquima extraxilar. Los tejidos secretores que rodean los canales y los tejidos de la vaina y del parénquima alrededor de los haces vasculares están reforzados con pectinas (tejidos colenquimáticos) de acuerdo con la tinción con rojo de rutenio (Fig. 2C). No se observó la presencia de alcaloides en la hoja de acuerdo con la prueba de Dragendorff.

Flor: El corte transversal del cáliz y corola muestra una epidermis uniestratificada, caracterizada por células de recubrimiento redondeadas de tamaños similares, sin estomas, ni tricomas. El mesófilo presenta varias capas de células del parénquima isodiamétricas, entre las cuales se observan los haces vasculares, uno central en sépalos y tres en pétalos. En cáliz y corola se presentan canales secretores de tipo A y tamaño menor a los de las hojas (Fig. 2J).

El filamento de los estambres presenta una epidermis uniestratificada, parénquima laxo y un haz vascular central (Fig. 2G). Las anteras son basifijas, presentan un exotecio (epidermis) uniestratificado, con contenido denso y oscuro, el endotecio uniestratificado es fibroso y está formado por células cuadrangulares, una capa muy delgada de estratos intermedios y un tapetum con contenido denso que se degrada en la medida que se conforman los granos de polen (Fig. 2D). La pared del gineceo presenta una epidermis externa, sin estomas, ni tricomas, conformada por células de recubrimiento redondeadas y con contenido denso (tanífero), debajo de la epidermis externa se observaron canales secretores de tipo A, esquizógenos y abundantes, debajo de los cuales el mesófilo es parenquimático, tanífero y con haces vasculares inmersos (Fig. 2E). En la parte inferior del ovario los bordes carpelares se fusionan en el centro y se presentan numerosos haces vasculares y parénquima laxo. Cada lóculo presenta dos filas de óvulos anátropos, siendo la placentación axilar. Sin embargo, en la parte superior del ovario los lóculos se unen y la placentación aparenta ser parietal (Fig. 2F).

En el análisis histoquímico, las pruebas de Sudán indican la presencia de aceites en los canales del cáliz, corola y ovario; también tiñen el polen y el estigma (Fig. 2H, Fig. 2I, Fig. 2J). Externamente las anteras dieron reacción positiva para sustancias fenólicas (cloruro férrico) (Fig. 2L) y para aceites (Sudán black) (Fig. 2K). El fluoroglucinol produce una coloración rosada-roja intensa en el xilema de los haces vasculares. La prueba de Dragendorff fue negativa para alcaloides. Los resultados anteriormente mencionados se encuentran resumidos en la tabla 1.

\section{DISCUSIÓN}

La estructura morfológica de los órganos de $H$. juniperinum debe entenderse en el contexto de las condiciones de vida en el páramo, de acuerdo con Mora-Osejo y Sturm (1995). En este trabajo los autores establecen las características que determinan xeromorfía o mesomorfía en 49 plantas de páramo, incluyendo Hypericum goyanesii, $H$. mexicanum y $H$. 

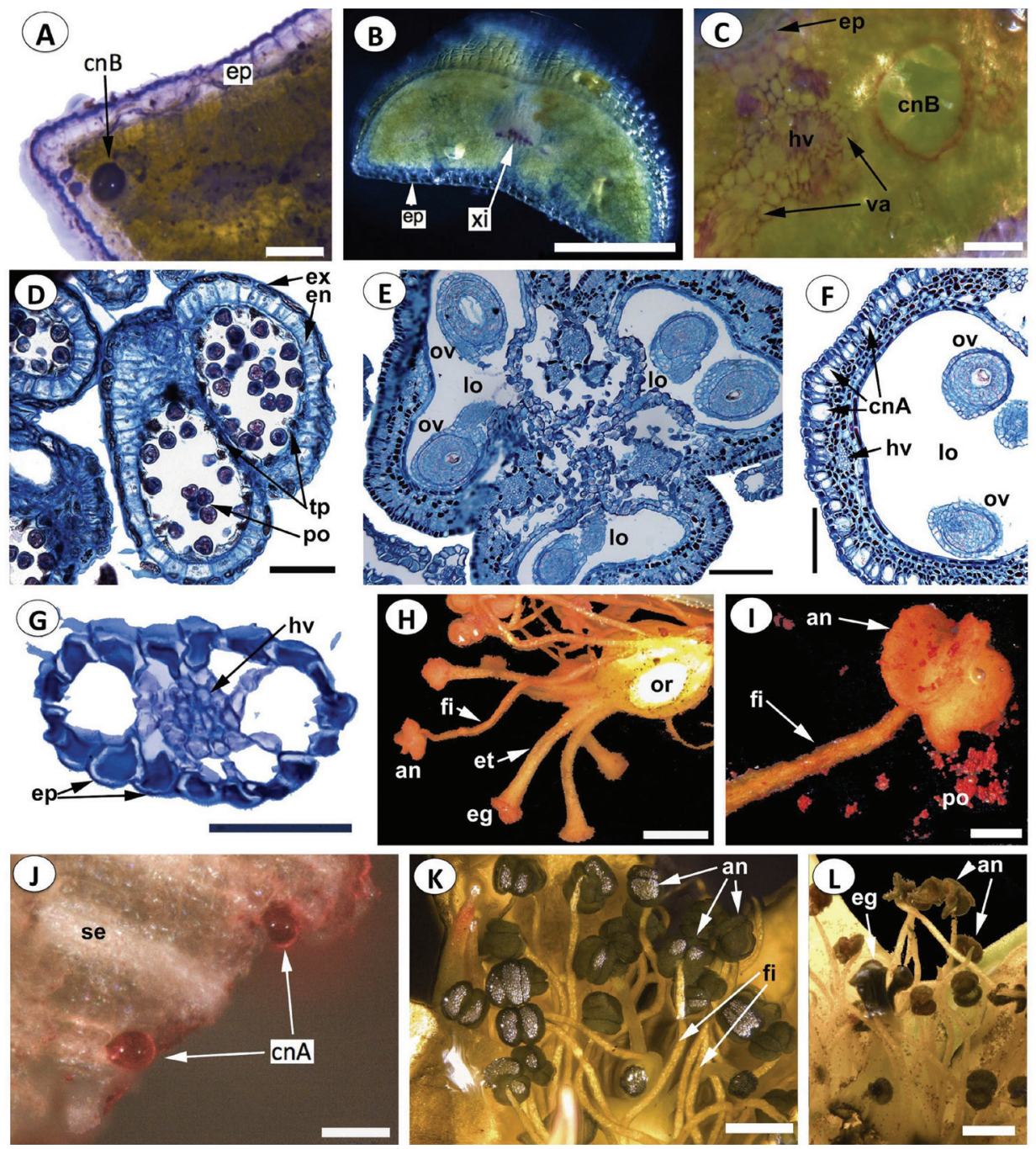

Fig. 2. Histoquímica foliar y anatomía e histoquímica floral de Hypericum juniperinum. A-C. Pruebas histoquímicas en hoja; A. Sudan black; B. Fluoroglucinol; C. Rojo de rutenio; D. Sección transversal (ST) de antera; E. ST del gineceo trilocular; F. ST de la pared del ovario; G. ST del filamento del estambre; H-L. Pruebas histoquímicas en flor; H. Sudan III en gineceo; I. Sudan III en anteras; J. Sudan III, positivo para los canales del sépalo; K. Sudan black en anteras; L. Prueba de cloruro férrico. $\mathrm{An}=$ antera, $\mathrm{CnA}=$ canales tipo $\mathrm{A}, \mathrm{cnB}=$ canales tipo $\mathrm{B}$, ep = epidermis, en = endotecio, ex = exotecio, eg $=$ estigma, et $=$ estilo, $\mathrm{fi}=$ filamento, $\mathrm{hv}=$ haz vascular, lo $=$ lóculo, or $=$ ovario, ov $=$ óvulo, po $=$ polen, $\mathrm{se}=$ sépalo, tp = tapetum, va = vaina haz vascular, xi = xilema. A-C., H-L. Estereomicroscopio; D-G. Microscopio óptico, tinción con astra blue-Fucsina básica. Escalas: $1 \mathrm{~mm}$ en H, K-L., $500 \mu \mathrm{m}$ en B., $200 \mu \mathrm{m}$ en I., $100 \mu \mathrm{m}$ en A, C, E, F, J., $50 \mu \mathrm{m}$ en D, G. Figuras a color en la versión digital.

Fig. 2. Foliar histochemistry and anatomy and floral histochemistry of Hypericum juniperinum. A.-C. Histochemical tests on leaves; A. Sudan black; B. fluoroglucinol; C. ruthenium red; D. Anther cross section (ST); E. ST of the trilocular gynoecium; F. ST of the ovary wall; G. ST of the filament of the stamen; H-L. Histochemical tests in flower; H. Sudan III in gynoecium; I. Sudan III in anthers; J. Sudan III, positive for sepal channels; K. Sudan black in anthers; L. Ferric chloride test. An = anther, $\mathrm{CnA}=$ type $\mathrm{A}$ channels, $\mathrm{cnB}=$ type $\mathrm{B}$ channels, ep = epidermis, en = endothecium, ex = exothecium, eg = stigma, et $=$ style, $\mathrm{fi}=$ filament, $\mathrm{hv}=$ vascular bundle, $\mathrm{lo}=$ loculus, or $=$ ovary, ov $=$ ovule, po $=$ pollen, $\mathrm{se}=$ sepal, $\mathrm{tp}=$ tapetum, va $=$ vascular sheath, xi = xylem. A-C., H-L. Stereomicroscope; D-G. Optical microscope, staining with astra blue-basic fuchsine basic. Scales: $1 \mathrm{~mm}$ in H, K-L., $500 \mu \mathrm{m}$ in B., $200 \mu \mathrm{m}$ in I., $100 \mu \mathrm{m}$ in A, C, E, F, J., $50 \mu \mathrm{m}$ in D, G. Colored figures in the digital version. 
TABLA 1

Resultados de las pruebas histoquímicas en los órganos aéreos de Hypericum juniperinum

\begin{tabular}{|c|c|c|c|c|c|}
\hline $\begin{array}{l}\text { Órgano de } \\
\text { Hypericum } \\
\text { juniperinum }\end{array}$ & Sudan IV y black & Cloruro férrico & Fluoroglucinol & Dragendorff & Rojo de rutenio \\
\hline TALLO & $\begin{array}{c}\text { Reacción en corteza } \\
\text { y floema }\end{array}$ & $\begin{array}{l}\text { Reacción en } \\
\text { médula y floema }\end{array}$ & $\begin{array}{c}\text { Reacción con } \\
\text { lignina del xilema }\end{array}$ & - & $\begin{array}{l}\text { Pectinas en } \\
\text { el xilema }\end{array}$ \\
\hline HOJA & $\begin{array}{l}\text { Cutícula de epidermis, } \\
\text { canales y parénquima }\end{array}$ & $\begin{array}{c}\text { Reacción en todos } \\
\text { los tejidos }\end{array}$ & $\begin{array}{l}\text { Reacción en xilema } \\
\text { del haz vascular }\end{array}$ & - & $\begin{array}{c}\text { Parénquima } \\
\text { alrededor } \\
\text { del haz vascular }\end{array}$ \\
\hline FLOR & $\begin{array}{l}\text { Canales, cáliz, corola, } \\
\text { ovario, anteras y polen }\end{array}$ & $\begin{array}{l}\text { Reacción en } \\
\text { las anteras }\end{array}$ & $\begin{array}{l}\text { Reacción en xilema } \\
\text { del haz vascular }\end{array}$ & - & - \\
\hline
\end{tabular}

strictum. De acuerdo con estas características, $H$. juniperinum es altamente xeromórfica al presentar hojas cartáceas, arrosetadas, convolutas y en posición vertical, con parénquima en empalizada biestratificado, paredes engrosadas, cutícula gruesa y cera epicuticular en las dos epidermis foliares, estomas en la cara abaxial y con cámara epiestomática. Sin embargo, presenta algunas características mesomórficas como el haz conductor con vaina parenquimática (no esclerenquimática) y parénquima esponjoso laxo.

Al realizar una comparación entre especies europeas de Hypericum (H. perforatum, $H$. perfoliatum, $H$. tetrapterum, $H$. androsaemum у $H$. hircinum) con Hypericum juniperinum se observaron similitudes como, la presencia de hojas convexas en la cara adaxial, epidermis monoestratificada y glándulas de tamaño variable distribuidas en el mesófilo (Perrone et al., 2013). Sin embargo; también se aprecian diferencias, puesto que las especies europeas presentan hojas dorsiventrales (bifaciales sensu Mora-Osejo \& Sturm, 1995) o isobilaterales (unifaciales sensu Mora-Osejo \& Sturm 1995), en tanto que las especies de Hypericum del páramo son equifaciales $(H$. goyanesii, $H$. mexicanum y $H$. juniperinum) o dorsiventrales inversas en $H$. strictum.

En el tallo de Hypericum juniperinum la presencia y disposición de los canales secretores en el floema (tipo A), el parénquima con células taníferas, y el xilema con porosidad difusa es similar a lo establecido por Perrone et al., (2013) para otras especies de Hypericum; tales como $H$. pubescens y $H$. aegypticum. Al comparar los resultados del presente estudio con los reportados por Schweingruber, Börner y Schulze (2011) para 16 especies de Hypericum, se encuentra que $H$. juniperinum no genera anillos anuales, pero si presenta gotas de aceite (oil drops) y canales secretores en el floema, al igual que las especies de Hypericum analizadas.

Con referencia a las diferentes estructuras secretoras, Hypericum juniperinum presenta canales tipo A en el ovario de la flor, así como en el floema del tallo, similar al estudio de Lotocka y Osinska (2010), y canales tipo B en el mesófilo de la hoja; canales que han sido reportados en otras especies del género Hypericum. Bottega, Garbari y Pagni (2004), destacan en Hypericum elodes la existencia de tres tipos de canales diferentes a lo largo de la flor, dependiendo de su ontogenia: en el pedúnculo floral canales de tipo $\mathrm{A}$, en los sépalos canales de tipo B y finalmente en la pared ovárica canales de tipo C. Adicionalmente, las pruebas histoquímicas realizadas para Hypericum juniperinum corroboran la presencia de metabolitos de tipo lipídico almacenados en estas estructuras.

Hypericum perforatum, especie ampliamente estudiada perteneciente a la sección Hypericum, presenta glándulas negras en hojas, flores y en mayor cantidad en los estambres (Zobayed, Freen, Goto, \& Koza, 2006). Este tipo de glándulas fueron definidas 
ontogenéticamente por Ciccarelli et al., (2001b) como agrupamientos de células secretoras rodeadas por una vaina de células aplanadas que en su madurez se llenan de contenido, principalmente hipericina y pseudohipericina (Zobayed et al., 2006). En $H$. juniperinum no se aprecian estas estructuras. Adicionalmente, al utilizar cromatografía en capa delgada con reveladores específicos, se confirma la ausencia de estos metabolitos en el extracto metanólico de esta especie (Mejía-Agudelo, 2017), lo que coincide con la descripción realizada por Crockett y Robson (2011) para la sección Brathys a la que pertenece $H$. juniperinum.

Por otra parte, $H$. juniperinum si presenta glándulas translúcidas, estructuras secretoras características de todo el género (Robson, 1987). Según Ciccarelli et al., (2001a) indican que este tipo de glándulas se encuentran distribuidas en la hoja, sépalos y pétalos. Por otro lado, Soelberg, Jorgensen y Ger (2007) encontraron en $H$. perforatum, especie empleada para el tratamiento de la depresión, que estas glándulas sintetizan el metabolito hiperforina, junto con algunos aceites esenciales que comparten la misma ruta biosintética. En el presente trabajo, a través del empleo de tinciones con Sudán, se confirma la presencia de sustancias de carácter lipídico alojadas en estas estructuras y que son importantes para la defensa de la planta frente a herbivoría (Ciccarelli et al., 2001a). Dicha defensa se da mediante la biosíntesis y almacenamiento de lípidos no volátiles, que están involucrados en la reducción de la alimentación por parte de los insectos o en la disrupción de los procesos de crecimiento de los mismos (Seigler, 1983).

El estudio histoquímico y anatómico permitió detectar ciertos metabolitos como aceites, fenoles y lignina en los tejidos de $H$. juniperinum. Adicionalmente no se detectaron alcaloides, lo que coincide con la revisión realizada por Crockett y Robson (2011). Por otro lado, lo referido en el análisis histoquímico concuerda con el trabajo realizado por Plazas (2017), que encontró que los metabolitos presentes en el extracto etanólico de Hypericum juniperinum son terpenos, esteroides, fenoles, taninos, flavonoides, quinonas y cumarinas, careciendo de glicósidos cardiotónicos y alcaloides. Lo anterior, junto con el artículo publicado por Mejía et al. (2019); permite una caracterización especifica de los flavonoides y sustancias fenólicas encontradas en los extractos de esta planta de interés medicinal que crece en los páramos colombianos.

El presente trabajo es el primer reporte de las principales características anatómicas e histoquímicas de Hypericum juniperinum. En conclusión, las características anatómicas principales en las hojas de $H$. juniperinum, la disposición en roseta, la presencia de glándulas translúcidas, los estomas en la cara adaxial, vaina de contenido tanífero y canales de tipo B; en el tallo ductos con 4 a 5 células secretoras, floema con radios, vasos uniperforados y punteaduras reticuladas, y finalmente en la flor no hay presencia de tricomas, canales secretores tipo A en cáliz y gineceo. Los resultados obtenidos relacionan la anatomía y sus adaptaciones estructurales con el tipo de vida que $H$. juniperinum presenta; y la necesidad de estas para su supervivencia en el páramo. Adicionalmente, la descripción macro y microscópica brindada para hojas, tallos y flores, así como la ubicación específica de los metabolitos en los tejidos de $H$. juniperinum, proveen información clave para la caracterización y autentificación de esta especie andina que tiene el potencial de ser utilizada como materia prima en la elaboración de productos útiles para el tratamiento de trastornos mentales como la depresión.

Declaración de ética: los autores declaran que todos están de acuerdo con esta publicación y que han hecho aportes que justifican su autoría; que no hay conflicto de interés de ningún tipo; y que han cumplido con todos los requisitos y procedimientos éticos y legales pertinentes. Todas las fuentes de financiamiento se detallan plena y claramente en la sección de agradecimientos. El respectivo documento legal firmado se encuentra en los archivos de la revista. 


\section{AGRADECIMIENTOS}

A la Universidad Nacional de Colombia, el DIB Bogotá con la CONVOCATORIA NACIONAL DE PROYECTOS PARA EL FORTALECIMIENTO DE LA INVESTIGACIÓN, CREACIÓN E INNOVACIÓN DE LA UNIVERSIDAD NACIONAL DE COLOMBIA 2016-2018, Cód: 34913.

\section{RESUMEN}

Hypericum es un género que pertenece a la familia Hypericaceae, conformado por 469 especies de hierbas, árboles y arbustos. Dos secciones en particular, Brathys y Trygynobrathys, son parte integral de la flora de Páramo. Hypericum tiene una amplia variedad de metabolitos que están asociados con múltiples actividades farmacológicas, y específicamente en $H$. juniperinum se han reportado propiedades antidepresivas relacionadas con la presencia de flavonoides y compuestos fenólicos. El objetivo de esta investigación fue relacionar las estructuras anatómicas con los metabolitos detectados por métodos histoquímicos presentes en los órganos vegetativos y reproductivos de Hypericum juniperinum recolectados en el Páramo colombiano. Los órganos aéreos (tallos, hojas y flores) de Hypericum juniperinum se recolectaron en la vereda Arbolocos del municipio de Cuitiva, Boyacá (código voucher No.589611, COL). Para el análisis anatómico, los órganos de $H$. juniperinum se procesaron con técnicas de fijación, incorporación en parafina, corte en microtomo y tinción con azul de astra y fucsina básica. Para el análisis histoquímico se realizaron cortes a mano alzada con tinciones específicas para lípidos, compuestos fenólicos, alcaloides y pectinas. La anatomía de los órganos de $H$. juniperinum debe entenderse en el contexto de las condiciones de vida del Páramo. Las hojas son altamente xeromorfas ya que son cartáceas, arrosetadas, convolutas y verticales, con parénquima en empalizada biestratificado, paredes engrosadas, cutícula gruesa y cera epicuticular en ambas epidermis foliares, estomas en el lado abaxial con cámara epidérmica. Sin embargo, tiene algunas características mesomórficas, como las vainas parenquimáticas de los haces vasculares y el parénquima esponjoso suelto. Hypericum juniperinum presenta glándulas translúcidas en hojas, cáliz y gineceo, y carece de las glándulas negras típicas de la sección Hypericum a la que pertenece la planta medicinal Hypericum perforatum. El análisis histoquímico mostró lípidos en los canales de la hoja, parénquima radial del tallo y en algunas estructuras florales. Los compuestos fenólicos se detectaron a lo largo de la hoja, en el floema del tallo y en las anteras. Hypericum juniperinum carece de alcaloides y presenta pectinas en el parénquima de la vaina que rodea el haz vascular de la hoja. El presente trabajo contribuye a relacionar los metabolitos con su distribución en los tejidos de esta especie nativa. Además, la anatomía mostró que $H$. juniperinum tiene características de una planta de Páramo. La descripción que se proporciona aquí podría utilizarse como una monografía preliminar de plantas del género Hypericum con propiedades terapéuticas prometedoras.

Palabras clave: caracterización anatómica; compuestos fenólicos; histoquímica; glándulas translúcidas; canales.

\section{REFERENCIAS}

Bottega, S., Garbari, F., \& Pagni, A. (2004). Hypericum elodes L. (Clusiaceae): The secretory structures of the flower. Israel Journal of Plant Sciences, 52(1), 51-57.

Brechu-Franco, A. E., Laguna-Hernández, G., De-la-CruzChacón, I., \& González-Esquinca, A. R., (2016). In situ histochemical localization of alkaloids and acetogenins in the endosperm and embryonic axis of Annona macroprophyllata Donn. Sm. seeds during germination. European Journal of Histochemistry, 60(1), 55-58.

Ciccarelli, D., Andreucci, A., \& Pagni, A. (2001a). Translucent glands and secretory canals in Hypericum perforatum L. (Hypericaceae): Morphological, anatomical and histochemical studies during the course of ontogenesis. Annals of Botany, 88(4), 637-644.

Ciccarelli, D., Andreucci, A., \& Pagni, A. (2001b). The "black nodules" of Hypericum perforatum L. subsp. perforatum: Morphological, anatomical, and histochemical studies during the course of ontogenesis. Israel Journal of Plant Sciences, 49(1), 33-40.

Crockett, S., Eberhardt, M., Kunert, O., \& Schübly, W. (2010). Hypericum species in the Paramos of Central and South America: a special focus upon H. irazuense Kunze ex N. Robson. Phytochemical Reviews, 9(2), 255-269.

Crockett, S. L., \& Robson, N. (2011). Taxonomy and chemotaxonomy of the genus Hypericum. Medicinal and Aromatic Plant Science and Biotechnology, 5(1), $1-13$.

Curtis, J. D., \& Lersten, N. R. (1990). Internal secretory structures in Hypericum (Clusiaceae): H. perforatum L. and H. balearicum L. New Phytologist, 114(4), 571-580.

Duarte, B., \& Parra, S. (2015). Plantas del páramo y sus usos para el buen vivir: Páramos de Guerrero y Rabanal. Bogotá, Colombia: Instituto de Investigación de Recursos Biológicos Alexander von Humboldt.

Ernst, E. (2003). Hypericum: The genus Hypericum. New York: Taylor \& Francis 
Green, J. R. (1884). On the organs of secretion in the Hypericaceae. Journal of the Linnean Society of London, Botany, 20(130), 451-464.

Greeson, J., Sanford, B., \& Monti, D. A. (2001). St. John's wort (Hypericum perforatum): a review of the current pharmacological, toxicological, and clinical literature. Psychopharmacology, 153(4), 402-414.

Johansen, D. A. (1940). Plant microtechnique. New York: McGraw-Hill.

Łotocka, B., \& Osinska, E. (2010). Shoot anatomy and secretory structures in Hypericum species (Hypericaceae). Botanical Journal of the Linnean Society, 163(1), 70-86

Marín, C., \& Parra, S. (2015). Bitácora de Flora: guía visual de plantas de páramos en Colombia. Bogotá: Instituto de Investigación de Recursos Biológicos Alexander von Humboldt.

Mejía-Agudelo, L. A. (2017) Estudio morfo-anatómico, fitoquímico y evaluación de la actividad sobre sistema nervioso central en modelo murino de Hypericum juniperinum (Kunth) (Tesis de Maestría). Sede Bogotá, Universidad Nacional de Colombia.

Mejía-Agudelo, L. A., Rojas, M. A., Guerrero-Pabón, M. F., Ramos, F. A., Castellanos, L., \& Marín-Loaiza, J. C. (2019). Antidepressant-like effects of methanol extract and fractions of Hypericum juniperinum Kunth in the forced swimming test. Pharmacognosy Magazine, 15(61), 226-231.

Mora-Osejo, L., \& Sturm, H. (1995). Estudios ecológicos del páramo y del bosque altoandino cordillera Oriental de Colombia (Tomo 1). Bogotá: Academia Colombiana de Ciencias Exactas, Fisicas y Naturales. Colección Jorge Alvarez Lleras $\mathrm{N}^{\circ} 6$.

Nürk, N. M. (2011). Phylogenetic analyses in St. John's wort (Hypericum): Inferring character evolution and historical biogeography (Doctoral dissertation). Freien Universität, Berlin, Germany.

Perrone, R., De Rosa, P., De Castro, O., \& Colombo, P. (2013). Leaf and stem anatomy in eight Hypericum species (Clusiaceae). Acta Botanica Croatica, 72(2), 269-286.

Plazas, E. (2017). Tamizaje fitoquímico y actividad antibacteriana in vitro de extractos y fracciones de tres especies colombianas del género Hypericum. Revista Cubana de Plantas Medicinales, 22(1), 1-14.

Robson, N. K. (1987). Studies in the genus Hypericum L. (Guttiferae): 7. Section 29. Brathys (part 1). Bulletin of the British Museum (Natural History) Botany, 16, 1-106. Retrieved from http://hypericum.myspecies. info/node/837

Robles, A., Raz, L., \& Marquínez, X. (2016). Anatomía floral de Peristethium leptostachyum (Loranthaceae). Revista de Biología Tropical, 64(1), 357-368.

Ruzin, S. (1999). Plant microtechnique and microscopy. New York: Oxford University Press.

Saddiqe, Z., Naeem, I., \& Maimoona, A. (2010). A review of the antibacterial activity of Hypericum perforatum L. Journal of Ethnopharmacology, 531(3), 511-521.

Schweingruber, F., Börner, A., \& Schulze, E. (2011). Atlas of stem anatomy in herbs, shrubs and trees (Vol. 1). New York: Springer.

Seigler, D. (1983). Role of Lipids in Plant Resistance to Insects. In P. Hedin (Ed.), Plant Resistance to Insects (pp. 303-327). Washington D.C: American Chemical Society.

Sirvent, T. M., Krasnoff, S. B., \& Gibson, D. M. (2003). Induction of hypericins and hyperforins in Hypericum perforatum in response to damage by herbivores. Journal of Chemical Ecology, 29(12), 2667-2681.

Soelberg, J., Jorgensen, L., \& Ger, A. (2007). Hyperforin accumulates in the translucent glands of Hypericum perforatum. Annals of Botany, 99(12), 1097-1100.

Stojanovic, G., Dordevic, A., \& Smelcerovic, A. (2013). Do other Hypericum species have medical potencial as St. John's wort (Hypericum perforatum)? Current Medicinal Chemistry, 20(18), 2273-2295.

Texeira, G., \& Monteiro, A. (2017). Leaf phytognostic characters of six species of Hypericum L. (Hypericaceae). Research and Analysis Journal of Applied Research, 3(12), 1303-1314.

Zobayed, S., Freen, F., Goto, E., \& Koza, T. (2006). PlantEnvironment interactions: Accumulation of hypericin in dark glands of Hypericum perforatum. Annals of Botany, 98(4), 793-804. 